\title{
Natural Incidence of Mixed Infections and Experimental Cross Protection Between Two Genotypes of Tobacco mild green mosaic virus
}

\author{
Sohrab Bodaghi, Deborah M. Mathews, and J. Allan Dodds
}

First author: Microbiology Program, and second and third authors: Department of Plant Pathology, University of California, Riverside 92521-0122.

Current address of S. Bodaghi: HIV and AIDS Malignancy Branch, Center for Cancer Research, NCI/NIH, 10 Center Dr., Rm. 10 S255

MSC 1868, Bethesda, MD 20892-1868.

Accepted for publication 10 August 2004.

\begin{abstract}
Bodaghi, S., Mathews, D. M., and Dodds, J. A. 2004. Natural incidence of mixed infections and experimental cross protection between two genotypes of Tobacco mild green mosaic virus. Phytopathology 94:1337-1341.

Isolates of Tobacco mild green mosaic virus (TMGMV), a member of the genus Tobamovirus, from Nicotiana glauca in southern California fall into two major genotypes, large (TMGMV-L) and small (TMGMV-S), distinguishable by the size of the coat protein $(\mathrm{CP})$ subgenomic RNA. Mixed infections in the field were rare (1.6\%), even at sites where both genotypes were common in single infections (62\% for TMGMV-S; $37 \%$ for TMGMV-L). When plants experimentally protected by TMGMV-L were challenged by TMGMV-S, almost complete cross protection $(90 \%$ of total plants challenged) was observed regardless of the protective time period (minimum $12 \mathrm{~h}$ and maximum 14 days). When plants protected by

TMGMV-S were challenged with TMGMV-L, complete cross protection was observed when the protective time was 5 to 14 days. However, when the protective time was 3 days or less, protection by TMGMV-S was greatly reduced $(11 \%)$, with mixed infections of TMGMV-S and -L predominating (69\%), and single infections of the challenge virus TMGMV$\mathrm{L}$ were frequently observed (20\%). When TMGMV-S and -L virions were co-inoculated, the virus progeny from individual plants most often contained only the TMGMV-L genome (61\%) or, less frequently (39\%), both genotypes. Therefore, TMGMV-L was more competitive than TMGMV-S and was able to displace TMGMV-S in experimental situations. The results obtained from cross-protection experiments in the greenhouse would explain the low frequency of natural mixed infections. It is possible that the experimental superior competitiveness of the novel L genotype has already or will play a role in its abundance in southern California.
\end{abstract}

Nicotiana glauca Grah. (tree tobacco) is a perennial wild plant known to harbor natural multiple infections of several different viruses, including Tobacco mild green mosaic virus (TMGMV), Satellite tobacco mosaic virus (STMV), Cucumber mosaic virus (CMV) with or without its satellite RNA CARNA 5, and Tobacco etch virus $(1,10)$. Genetic diversity of TMGMV from several countries has been described (15). Two different genotypes of TMGMV, large (TMGMV-L) and small (TMGMV-S), have been characterized from $N$. glauca plants in southern California that differ in the length of the $3^{\prime}$ untranslated region (UTR) of the single-stranded RNA genome (2). The difference in length is the result of a repeated sequence (147 nucleotides) that incorporates the three pseudoknots found in the 3' UTR of TMGMV-S. The two genotypes also express some biological differences when inoculated onto a variety of host plants. Interactions between these two genotypes of TMGMV have not been studied.

Prior to our recent analysis of California isolates of TMGMV (2), the existence of different genotypes of a single Tobamovirus species having $3^{\prime}$ UTRs with significantly different sizes was not suspected. The discovery of both genotypes in a single area, southern California, presented an opportunity to study interactions between genome variants of a single virus in a natural population. The objectives of this initial study were to determine the frequency of single and mixed infections of the two genotypes in southern California, as well as the effects of protecting time, host species, and leaf position used for challenge inoculations on cross protection. The role cross protection might play in the pre-

Corresponding author: D. M. Mathews; E-mail address: dmathews@ucr.edu

Publication no. P-2004-1011-03R

(c) 2004 The American Phytopathological Society sent and future distribution of the expected (three-pseudoknot) and novel (six-pseudoknot) genotypes of TMGMV in a natural host, $N$. glauca, is discussed.

\section{MATERIALS AND METHODS}

Double-stranded RNA analysis. Double-stranded (ds)RNA was isolated from $7 \mathrm{~g}$ of leaf tissue from infected plants and purified by two cycles of CF-11 cellulose chromatography as previously described (5). dsRNAs were analyzed using $6 \%$ polyacrylamide gel electrophoresis and stained with ethidium bromide at $100 \mathrm{ng} / \mathrm{ml}$.

Natural incidence of mixed infections. To investigate the natural incidence of mixed infections, a polymerase chain reaction (PCR) approach was used with the expectation that two DNA fragments of different sizes would be generated from a mixed infection. dsRNA from original field samples $(5 \mu \mathrm{l}=100$ to $200 \mathrm{ng})$ was denatured for use as target RNA in reverse-transcriptase (RT)-PCR reactions by boiling for $5 \mathrm{~min}$ in $10 \mu \mathrm{l}$ of $\mathrm{H}_{2} \mathrm{O}$ and snap chilling on ice. Avian myeloblastosis virus RT (AMV-RT; Promega Corp., Madison, WI) directed the synthesis of first-strand cDNA using the denatured dsRNA and a $3^{\prime}$ end-specific primer, (5'CGTGAATTCACCGGTTGGGCCGCTACCCGCGGTTA3' = $3^{\prime}$ end primer), which included 20 bases (underlined) complementary to the $3^{\prime}$ end of both TMGMV genotypes (2) and an EcoRI restriction site. PCR was used to convert cDNAs to dsDNA forms by using a second-strand primer (5'ATAGAATTCGCGGCCGCTCAATATGCCTTATACAATCAACTCTCCGA3' = coat protein $[\mathrm{CP}] 5^{\prime}$ end primer), containing an EcoRI site and sequences of the TMGMV CP gene from nucleotide 5661 to 5690 (underlined) (18). PCR reactions using Taq DNA polymerase (Promega Corp.) consisted of an initial denaturation for $2 \mathrm{~min}$ at $94^{\circ} \mathrm{C}$; 
followed by 30 cycles of $1 \mathrm{~min}$ at $94^{\circ} \mathrm{C}, 30 \mathrm{~s}$ at $60^{\circ} \mathrm{C}$, and $45 \mathrm{~s}$ at $72^{\circ} \mathrm{C}$; and then $5 \mathrm{~min}$ at $72^{\circ} \mathrm{C}$. dsRNA extractions from experimental single and mixed infections of $N$. glauca were used as controls and for RT-PCR amplification. RT-PCR products were subjected to electrophoresis in a $1 \%$ agarose gel and the sizes of the amplicons were compared.

Hosts, viruses, and inoculations. Hosts for cross-protection experiments were $N$. tabacum L. 'Xanthi' and $N$. glauca, which are systemic hosts for both genotypes of TMGMV. Plants were mechanically inoculated with $50 \mu \mathrm{l}$ of a $5 \mu \mathrm{g} / \mathrm{ml}$ suspension of gradient purified virus for each of the two TMGMV genotypes and maintained under standard greenhouse conditions.

Cross-protection and co-inoculation experiments. Treatments consisted of plants that were inoculated as follows: protected, but not challenged; challenged, but not protected; protected, then challenged; and mock inoculated. Inoculations were made to two to eight plants each of $N$. tabacum and $N$. glauca for

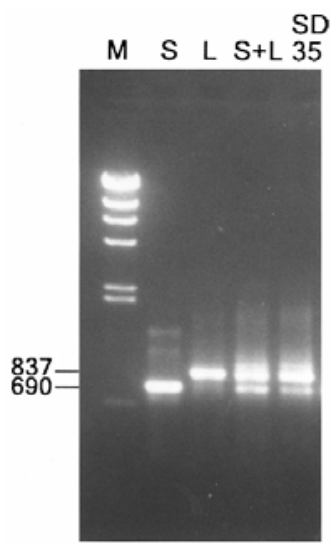

Fig. 1. Agarose gel electrophoresis of reverse-transcriptase polymerase chain reaction (RT-PCR) products from single and mixed infections of Tobacco mild green mosaic virus (TMGMV) small (TMGMV-S) and large (TMGMV-L). Double-stranded RNA targets used for RT-PCR were: S = TMGMV-S alone, field isolate R-1 \#22; L = TMGMV-L alone, field isolate R-1 \#18; S+L = experimental mixed infection of both types; SD-35 = field sample San Diego $35 ; \mathrm{M}=\lambda$ DNA digested with HindIII. Sizes (in base pairs) of the large (837) and small (690) amplicons are shown on the left margin.

TABLE 1. Identification of Tobacco mild green mosaic virus (TMGMV) genotypes present in field samples of Nicotiana glauca from various locations within southern California

\begin{tabular}{lcc}
\hline Geographic location $^{\text {a }}$ & TMGMV type $^{\mathrm{b}}$ & RT-PCR of field tissue $^{\mathrm{c}}$ \\
\hline SA & $\mathrm{L}$ & 4 \\
& S & 0 \\
SB & L & 5 \\
& S & 12 \\
SD & L & 3 \\
& S & 8 \\
& L+S & 1 \\
R-1 & L & 6 \\
& S & 4 \\
R-3 & L & 2 \\
& S & 15 \\
R-4 & L & 3 \\
& S & 0 \\
Totals & L & 23 \\
& S & 39 \\
& L+S & 1
\end{tabular}

${ }^{a}$ Isolates were collected from Santa Anita (SA), Santa Barbara (SB), San Diego (SD), and three different locations in Riverside (R1, R3, and R4).

${ }^{b}$ Designated as TMGMV-L (large, L), TMGMV-S (small, S), or mixed infections of TMGMV-L and $-\mathrm{S}(\mathrm{L}+\mathrm{S})$ based on reverse-transcriptase polymerase chain reaction (RT-PCR) analysis.

${ }^{c}$ Number of each TMGMV genotype based on RT-PCR of double-stranded RNAs extracted directly from field tissue. each treatment. Challenge inoculations were made to leaf positions one leaf above that of the protection inoculation unless otherwise noted. Leaves that became systemically infected were harvested 2 weeks after the last inoculation was performed. Challenge inoculations were done at 3, 5, 7, 10, and 14 days after the protecting inoculation as well as at 12 to $72 \mathrm{~h}$ (at 12-h intervals) after the protecting inoculation. Tobacco plants also were mechanically co-inoculated either on one leaf with a mixture of virions (1:1 ratio) of both TMGMV genotypes or simultaneously on two different leaves, with each individual genotype as described above.

\section{RESULTS}

Incidence of mixed infections in field samples of $N$. glauca. The original dsRNA samples from field tissue were used for RT-PCR. The PCR products were screened by agarose gel electrophoresis for fragments of the expected lengths for TMGMV-S (690 bp) and TMGMV-L (837 bp). Both 690- and 837-bp fragments were detected in samples from a plant experimentally doubly infected (Fig. 1, S+L). Isolate SD-35 from San Diego was the only field sample out of 63 field samples tested that generated both fragments, implying a mixed infection of both genotypes of TMGMV (Fig. 1, SD-35). The remaining 62 samples were identified as single infections of either TMGMV-S (39 samples) or TMGMV-L (23 samples) (Table 1).

dsRNA profiles from experimental plants singly inoculated with TMGMV-S and -L. Both TMGMV genotypes cause mild green mosaic symptoms in N. tabacum and are almost symptomless in N. glauca; therefore, neither TMGMV genotype can be distinguished by host reaction in experimental single infections. The relative electrophoretic mobilities of dsRNAs from each type of TMGMV are illustrated in Figure 2. Electrophoretic mobilities of the replicative form (RF) of both genotypes are similar. In this study, the CP subgenomic (sg) RNA was used as a primary marker to detect single or mixed infections and to analyze interactions between the two types of TMGMV-S and -L. In single infections (used as controls for cross-protection experiments in next section), the TMGMV-S CPsg dsRNA migrated considerably faster in the polyacrylamide gel than the equivalent subgenomic

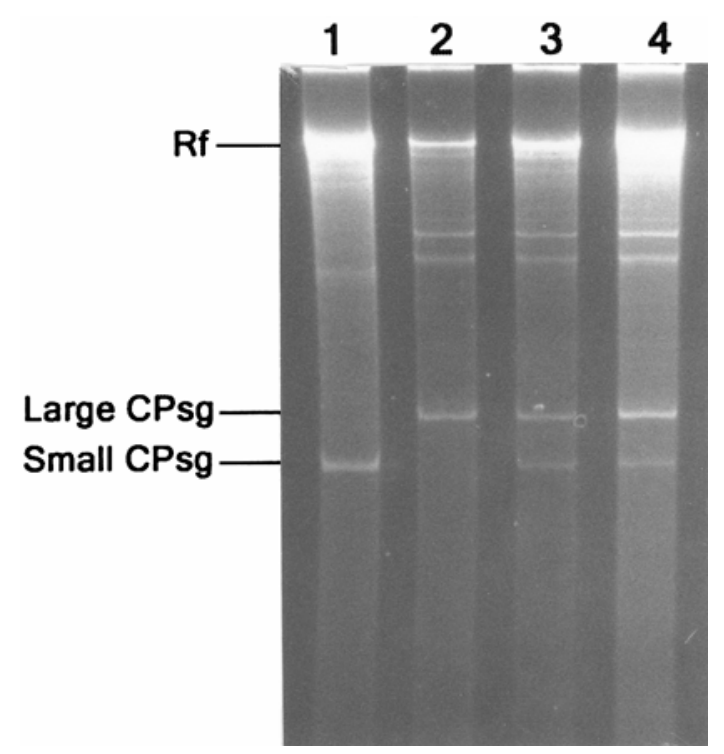

Fig. 2. Polyacrylamide gel electrophoresis of double-stranded (ds)RNAs extracted from Tobacco mild green mosaic virus (TMGMV) isolates with coat protein subgenomic (CPsg) regions designated as small (TMGMV-S, field isolate R-1 \#22, lane 1), large (TMGMV-L, field isolate R-1 \#18, lane 2), or mixed infections of both TMGMV-S and $-\mathrm{L}$ (lanes 3 and 4). The relative positions of each CPsg and replicative form (RF) dsRNA are shown on the left margin. 
dsRNA of TMGMV-L (Fig. 2, lanes 1 and 2, respectively). This shift in electrophoretic mobility corresponds to the difference in 3' UTR length between these two genotypes. dsRNA profiles of either genotype were similar in extracts from $N$. glauca and $N$. tabacum. However, there were some minor dsRNA bands associated with $N$. tabacum that were not present in $N$. glauca (data not shown). No indications of mixed infections were observed in singly inoculated plants. All other control plants (buffer and healthy sap inoculations) gave negative dsRNA results, as expected.

Initial cross-protection experiments with protection intervals of 3 days or more. The time interval between the protective inoculation and the challenge inoculation was $3,5,7,10$, or 14 days in initial experiments. No detectable accumulation of challenge virus CPsg dsRNA was observed for either of the two genotypes when the protective time was 5 days or more for both host plants (Table 2). TMGMV-L also was able to completely prevent the accumulation of TMGMV-S CPsg dsRNA when the protective interval was 3 days. However, when TMGMV-S was used as the protecting isolate for 3 days, TMGMV-L was able to overcome cross protection in a few plants of both hosts (Table 2). The dsRNA analysis from one of these plants is shown in Figure 2 , lane 3 . Based on these initial results, the only protective time which allowed superinfection by the challenging genotype was 3 days. Therefore, for the rest of the experiments, the protective time ranged from 12 to $72 \mathrm{~h}$ ( 3 days).
Cross-protection experiments with protection intervals of 3 days or less. Challenge inoculation on same leaf as protection inoculation. When plants protected by TMGMV-S were challenged by TMGMV-L on the same leaf, mixed infections were detected by dsRNA analysis in all $N$. tabacum plants regardless of the protective time period (minimum $12 \mathrm{~h}$, maximum $72 \mathrm{~h}$ ) (Table 3 , treatment 1). N. glauca showed different results during these time periods (12 to $72 \mathrm{~h}$ ). Of 12 plants, 3 were protected efficiently by TMGMV-S, 5 of 12 plants were infected with both genotypes, and, in 4 of 12 plants, only TMGMV-L was detected by dsRNA analysis (Table 3).

When $N$. tabacum was protected by TMGMV-L and challenged by TMGMV-S during the same time intervals, 10 of 12 plants were fully protected by TMGMV-L and 2 of 12 plants showed a mixed infection of both genotypes (Table 3, treatment 1; Fig. 2, lane 4). Complete cross protection was observed in $N$. glauca when plants protected by TMGMV-L were challenged by TMGMV-S, regardless of the protective time (minimum $12 \mathrm{~h}$ ) (Table 3).

Challenge inoculations on leaves above the position of protection inoculations. When plants protected by TMGMV-S were challenged by TMGMV-L on upper leaves, mixed infections were detected in all $N$. tabacum plants, the same result as when inoculations were on the same leaf (Table 3, treatment 2). However, in $N$. glauca, 1 plant was fully protected by TMGMV-S,

TABLE 2. Detection of Tobacco mild green mosaic virus (TMGMV) small (TMGMV-S) and large (TMGMV-L) by double-stranded RNA analysis of tobacco plants (Nicotiana spp.) used in cross-protection experiments with protecting times of 3 to 14 days

\begin{tabular}{|c|c|c|c|c|}
\hline \multirow[b]{2}{*}{ Inoculation schemes ${ }^{\mathrm{a}}$} & \multicolumn{2}{|c|}{ Experiment 1} & \multicolumn{2}{|c|}{ Experiment 2} \\
\hline & N. tabacum & N. glauca & N. tabacum & N. glauca \\
\hline Mock & $0 / 2$ & $0 / 2$ & $0 / 2$ & $0 / 2$ \\
\hline $\mathrm{S}$ & $7 / 7 \mathrm{~S}$ & $7 / 7 \mathrm{~S}$ & $5 / 5 \mathrm{~S}$ & $5 / 5 \mathrm{~S}$ \\
\hline $\mathrm{S}(3) \mathrm{L}$ & $2 / 2 \mathrm{~S}$ & $2 / 2 \mathrm{~S}+\mathrm{L}$ & $1 / 2 \mathrm{~S} ; 1 / 2 \mathrm{~S}+\mathrm{L}$ & $2 / 2 \mathrm{~S}$ \\
\hline $\mathrm{S}(5) \mathrm{L}$ & $2 / 2 \mathrm{~S}$ & $2 / 2 \mathrm{~S}$ & $2 / 2 \mathrm{~S}$ & $2 / 2 \mathrm{~S}$ \\
\hline$S(7) L$ & $2 / 2 \mathrm{~S}$ & $2 / 2 \mathrm{~S}$ & NA & NA \\
\hline $\mathrm{L}(5) \mathrm{S}$ & $2 / 2 \mathrm{~L}$ & $2 / 2 \mathrm{~L}$ & $2 / 2 \mathrm{~L}$ & $2 / 2 \mathrm{~L}$ \\
\hline $\mathrm{L}(7) \mathrm{S}$ & $2 / 2 \mathrm{~L}$ & $2 / 2 \mathrm{~L}$ & NA & NA \\
\hline $\mathrm{L}(10) \mathrm{S}$ & $2 / 2 \mathrm{~L}$ & $2 / 2 \mathrm{~L}$ & NA & NA \\
\hline $\mathrm{L}(14) \mathrm{S}$ & $2 / 2 \mathrm{~L}$ & $2 / 2 \mathrm{~L}$ & NA & NA \\
\hline
\end{tabular}

a $\mathrm{S}=$ TMGMV-S; $\mathrm{L}=$ TMGMV-L; $\mathrm{S}+\mathrm{L}=$ mixed infection of TMGMV-S and $-\mathrm{L} ; \mathrm{S}(X) \mathrm{L}=$ TMGMV-S-inoculated plants were challenge inoculated with TMGMV$\mathrm{L}$ on the leaf above the initial inoculation, where $X$ equals $3,5,7,10$, or 14 days later; and $\mathrm{L}(X) \mathrm{S}=\mathrm{TMGMV}$-L-inoculated plants were challenge inoculated with TMGMV-S on the leaf above the initial inoculation, where $X$ equals $3,5,7,10$, or 14 days later.

TABLE 3. Detection of Tobacco mild green mosaic virus (TMGMV) small (TMGMV-S) and large (TMGMV-L) by double-stranded RNA analysis of crossprotection experiments comparing Nicotiana spp. leaf position used for challenge inoculations with protecting times of 12 to $72 \mathrm{~h}$

\begin{tabular}{|c|c|c|c|c|}
\hline \multirow[b]{2}{*}{ Inoculation schemes ${ }^{\mathrm{a}}$} & \multicolumn{2}{|c|}{ Treatment 1 (inoculated leaf) } & \multicolumn{2}{|c|}{ Treatment 2 (upper leaf) } \\
\hline & N. tabacum & N. glauca & N. tabacum & N. glauca \\
\hline Mock & $0 / 4$ & $0 / 4$ & $0 / 4$ & $0 / 4$ \\
\hline $\mathrm{S}$ & $8 / 8 \mathrm{~S}$ & $8 / 8 \mathrm{~S}$ & $8 / 8 \mathrm{~S}$ & $8 / 8 \mathrm{~S}$ \\
\hline $\mathrm{S}(12) \mathrm{L}$ & $2 / 2 \mathrm{~S}+\mathrm{L}$ & $2 / 2 \mathrm{~L}$ & $4 / 4 \mathrm{~S}+\mathrm{L}$ & $3 / 4 \mathrm{~L} ; 1 / 4 \mathrm{~S}+\mathrm{L}$ \\
\hline$S(24) L$ & $2 / 2 \mathrm{~S}+\mathrm{L}$ & $1 / 2 \mathrm{~S} ; 1 / 2 \mathrm{~S}+\mathrm{L}$ & $4 / 4 \mathrm{~S}+\mathrm{L}$ & $2 / 4 \mathrm{~L} ; 2 / 4 \mathrm{~S}+\mathrm{L}$ \\
\hline $\mathrm{S}(36) \mathrm{L}$ & $2 / 2 \mathrm{~S}+\mathrm{L}$ & $2 / 2 \mathrm{~L}$ & 4/4 S+L & $2 / 4 \mathrm{~L} ; 2 / 4 \mathrm{~S}+\mathrm{L}$ \\
\hline $\mathrm{L}(12) \mathrm{S}$ & $2 / 2 \mathrm{~L}$ & $2 / 2 \mathrm{~L}$ & $4 / 4 \mathrm{~S}+\mathrm{L}$ & $4 / 4 \mathrm{~L}$ \\
\hline $\mathrm{L}(24) \mathrm{S}$ & $2 / 2 \mathrm{~S}+\mathrm{L}$ & $2 / 2 \mathrm{~L}$ & $2 / 4 \mathrm{~L} ; 2 / 4 \mathrm{~S}+\mathrm{L}$ & $4 / 4 \mathrm{~L}$ \\
\hline $\mathrm{L}(36) \mathrm{S}$ & $2 / 2 \mathrm{~L}$ & $2 / 2 \mathrm{~L}$ & $4 / 4 \mathrm{~L}$ & $4 / 4 \mathrm{~L}$ \\
\hline $\mathrm{L}(48) \mathrm{S}$ & $2 / 2 \mathrm{~L}$ & $2 / 2 \mathrm{~L}$ & 3/4 L; 1/4 S+L & $3 / 4 \mathrm{~L} ; 1 / 4 \mathrm{~S}+\mathrm{L}$ \\
\hline $\mathrm{L}(60) \mathrm{S}$ & $2 / 2 \mathrm{~L}$ & $2 / 2 \mathrm{~L}$ & $4 / 4 \mathrm{~L}$ & $4 / 4 \mathrm{~L}$ \\
\hline $\mathrm{L}(72) \mathrm{S}$ & $2 / 2 \mathrm{~L}$ & $2 / 2 \mathrm{~L}$ & $4 / 4 \mathrm{~L}$ & $4 / 4 \mathrm{~L}$ \\
\hline
\end{tabular}

${ }^{a} \mathrm{~S}=$ TMGMV-S; L = TMGMV-L; S+L = mixed infection of TMGMV-S and -L; S $(X) \mathrm{L}=$ TMGMV-S-inoculated plants were challenge inoculated with TMGMV$\mathrm{L}$ either on the same leaf or on the leaf above the initial inoculation, where $X$ equals $12,24,36,48,60$, or $72 \mathrm{~h}$ later; $\mathrm{L}(X) \mathrm{S}=\mathrm{TMGMV}$-L-inoculated plants were challenge inoculated with TMGMV-S either on the same leaf or on the leaf above the initial inoculation, where $X$ equals $12,24,36,48,60$, or $72 \mathrm{~h}$ later. 
while 11 of 24 plants showed mixed infections. In 12 of 24 plants, only TMGMV-L infection was detected (Table 3), again indicating that superinfections with TMGMV-L can displace TMGMV-S.

When TMGMV-L-protected $N$. tabacum plants were challenged by TMGMV-S, 17 of 24 were fully protected by TMGMV$\mathrm{L}$ and 7 of 24 plants showed mixed infections of both types (Table 3 ). It should be noted that 6 of the 7 plants showing mixed infections were observed at the 12- and 24-h intervals. With the exception of a mixed infection in a single plant (at $48 \mathrm{~h}$ after protection), complete cross protection again was observed in $N$. glauca regardless of the protective time period.

An additional observation in those plants that became infected with both genotypes was an increase in the severity of symptoms seen. Either genotype alone caused mild or no symptoms in $N$. tabacum or $N$. glauca, respectively. However, when both genotypes were present, $\approx 70 \%$ of the plants from both species showed symptoms such as leaf distortion, mottling, and moderate to severe mosaic.

Co-inoculation experiments. dsRNA analysis of plants co-inoculated with both types of TMGMV showed that 4 of $8 \mathrm{~N}$. tabacum plants and 7 of $20 \mathrm{~N}$. glauca plants had a mixture of both TMGMV-S and -L CPsg dsRNAs, whereas 4 of $8 \mathrm{~N}$. tabacum plants and 13 of $20 \mathrm{~N}$. glauca plants had only the TMGMV-L dsRNA (Table 4). No plants of either species had only the TMGMV-S CPsg dsRNA. Plants containing both genotypes showed a similar increase in symptom severity as seen in the cross-protection experiments.

To determine if the compositions of these populations would remain stable and to provide a source of "mixed dsRNA" for controls in RT-PCR reactions, six $N$. glauca plants infected with both genotypes were kept in the greenhouse and subjected to additional dsRNA analyses at 30, 45, and 60 days postinoculation (dpi). dsRNA results at 30 dpi still showed all six plants to be co-infected. However, at $45 \mathrm{dpi}$, only TMGMV-L CPsg was present in all six plants, with no TMGMV-S CPsg being detected. The same result was obtained when dsRNA analysis was performed at $60 \mathrm{dpi}$.

\section{DISCUSSION}

Interactions between the two genotypes were followed in experimental and natural infections of plants through the use of dsRNA and RT-PCR analyses. The dsRNA method was easy to use and interpret and was the method of choice for the experimental studies. Field-infected plants contained several other viruses in addition to TMGMV (4); therefore, a specific method (RT-PCR) was needed to estimate the frequency of natural single and mixed infections. Of 212 trees surveyed, $63 \mathrm{~N}$. glauca trees (30\%) from a variety of locales in southern California were positive for the presence of TMGMV. This is a lower incidence when compared with previous reports of TMGMV infecting $N$. glauca in Australia and Spain where average incidence was $>75 \%(14,15)$. Only 1 of the 63 plants positive for TMGMV $(1.6 \%)$ had a mixed infection of both genotypes. This frequency is similar to that found for mixed infections of Wheat streak mosaic virus in Nebraska $(2.3 \%)$, where 32 distinct genotypes were found (11). There was no obvious division of the two genotypes between locations; however, TMGMV-S accounted for $63 \%$ of the total. In two locations with small sample sizes, only TMGMV-L was found (Santa Anita and one of the three Riverside sites), and no sites contained only TMGMV-S. In three other sites where both genotypes were found, TMGMV-S was predominant (75\% of total on average). Bald and Goodchild (1) did not report a frequency of tobamovirus infection in their limited survey of $N$. glauca trees in the 1950s, but did find several tobamovirus variants that caused widely variable symptoms from different locations in southern California.

The low incidence of natural mixed infections of TMGMV-S and $-\mathrm{L}$, even in areas where both were present in close proximity, could be explained if these two types were able to cross protect against each other. Hall et al. (9) found that cross protection was one of three mechanisms responsible for genetic isolation between closely related strains of WSMV. The experimental studies on cross protection described here investigated the effects of various delayed inoculations and co-inoculations on the ability of the two TMGMV genotypes to systemically infect tobacco and $N$. glauca plants. When five or more days were allowed between protecting and challenge inoculations, the progeny populations were composed solely of the protecting virus and lacked detectable dsRNA accumulation of the challenging virus. This interaction is a clear example of the cross-protection phenomenon commonly reported between closely related plant viruses $(3,12,13,16,17)$. Similar results in $N$. tabacum and $N$. glauca indicate that TMGMV cross protection was not dependent on the host.

Experiments with different delays between protecting and challenge inoculation showed that longer delays, presumably providing for better establishment or spread of the protecting virus, resulted in more efficient exclusion of the challenge virus. These results are typical for cross-protection studies, but are discussed here to point out that the strong reciprocal results reported here for TMGMV may explain the observed predominance of single infections in the natural host $N$. glauca. The assumption here is that plants in the field probably will have at least 3 days to become infected with one genotype of TMGMV before superinoculation. Mechanical transmission is assumed to be responsible for natural transmission in the absence of any known natural vector, although distribution patterns of closely related isolates of TMGMV in Australia indicate the possible existence of an airborne vector (14).

Several observations lead to the conclusion that TMGMV-L is more competitive than TMGMV-S. When the delay between inoculation times is 3 days or less, TMGMV-L-protected plants demonstrated strong cross protection in N. glauca. Some exception was seen in $N$. tabacum, where a small number of plants became doubly infected, especially when an upper leaf position was used for the challenge inoculum. The overall results indicate that TMGMV-S has little propensity to overcome cross protection induced by TMGMV-L. However, when TMGMV-S-protected plants were examined, mixed infections of both types were found

TABLE 4. Detection of Tobacco mild green mosaic virus (TMGMV) small (TMGMV-S) and large (TMGMV-L) by double-stranded RNA analysis of tobacco plants (Nicotiana spp.) singly or co-inoculated with both genotypes of TMGMV ${ }^{a}$

\begin{tabular}{|c|c|c|c|c|c|c|c|}
\hline \multirow[b]{3}{*}{ Inoc. schemes ${ }^{b}$} & & & \multicolumn{4}{|c|}{ Experiment 2} & \multirow{3}{*}{$\frac{\text { Exp. } 3 \text { (inoc. leaf) }}{\text { N. glauca }}$} \\
\hline & \multicolumn{2}{|c|}{ Exp. 1 (inoc. leaf) } & \multicolumn{2}{|c|}{ Treatment 1 (inoc. leaf) } & \multicolumn{2}{|c|}{ Treatment 2 (upper leaf) } & \\
\hline & N. tabacum & N. glauca & N. tabacum & N. glauca & N. tabacum & N. glauca & \\
\hline Mock & $0 / 1$ & $0 / 1$ & $0 / 1$ & $0 / 1$ & $0 / 1$ & $0 / 1$ & $0 / 2$ \\
\hline $\mathrm{L}$ & $1 / 1 \mathrm{~L}$ & $1 / 1 \mathrm{~L}$ & $1 / 1 \mathrm{~L}$ & $1 / 1 \mathrm{~L}$ & $1 / 1 \mathrm{~L}$ & $1 / 1 \mathrm{~L}$ & $2 / 2 \mathrm{~L}$ \\
\hline $\mathrm{S}+\mathrm{L}$ & $4 / 4 \mathrm{~L}$ & $1 / 4 \mathrm{~S}+\mathrm{L} ; 3 / 4 \mathrm{~L}$ & $2 / 2 \mathrm{~S}+\mathrm{L}$ & $2 / 2 \mathrm{~L}$ & $2 / 2 \mathrm{~S}+\mathrm{L}$ & $2 / 2 \mathrm{~L}$ & $6 / 12 \mathrm{~S}+\mathrm{L} ; 6 / 12 \mathrm{~L}$ \\
\hline
\end{tabular}

${ }^{\text {a }}$ Exp. = experiment, inoc. $=$ inoculated.

b $\mathrm{S}=$ TMGMV-S; L = TMGMV-L; S+L = co-inoculated with TMGMV-S and -L simultaneously on the same or upper leaf and resulting mixed infections of both genotypes. 
in most plants by dsRNA analysis, especially in $N$. tabacum. Therefore, TMGMV-L has a propensity to overcome cross protection induced by TMGMV-S. This trend was observed regardless of leaf position used for challenge inoculations on N. tabacum. Further analysis of the data showed that TMGMV-L can displace TMGMV-S, even when TMGMV-S was used as the protective inoculation. These examples were all in N. glauca, at the 12- and 36-h intervals when both inoculations were on the same leaf, and at the 12- to 72-h intervals (except $48 \mathrm{~h}$ ) for plants challenged on an upper leaf (Table 3). Therefore, the cross protection conferred by TMGMV-S is not as efficient as that of TMGMV-L.

The dsRNA patterns clearly showed mixtures of both TMGMV$\mathrm{S}$ and $-\mathrm{L}$ in some experimental plants, including those that were co-inoculated. Thus, the two genotypes can co-replicate in both tobacco species tested. TMGMV-L alone, however, was detected in the majority of co-inoculated plants, indicating that TMGMV-L predominates in the resulting infections despite co-inoculation of equal amounts of inocula. The dsRNA pattern for TMGMV-S alone (without TMGMV-L) was not recovered from any tobacco plants during co-inoculation experiments, indicating that TMGMV-S cannot efficiently compete with TMGMV-L and may commonly be eliminated from mixed infections.

When doubly infected plants were resampled to assess the composition of the population, only TMGMV-L was isolated at 45 and 60 dpi. One explanation for these results is that TMGMV-S populations, through RNA recombination, became TMGMV-L; therefore, only TMGMV-L was detected using dsRNA. Another interpretation is that TMGMV-L causes a decrease of TMGMV-S populations below a threshold at which TMGMV-S can be detected by dsRNA through competitive exclusion $(6,8)$. A similar phenomenon might have caused the disappearance of Tobacco mosaic virus and the establishment of TMGMV in N. glauca during the last century in Australia (7).

A general conclusion from this study is that TMGMV-L with six pseudoknots is more competitive than TMGMV-S with three pseudoknots in experimental mixed infections. It is possible that the cross protection observed between these genotypes, together with the competitive advantage that TMGMV-L possesses, explains the paucity of mixed infections in the field. If it is further assumed that the novel six-pseudoknot form, which is known only in southern California, is a more recent introduction into $N$. glauca, it is possible that TMGMV-L is in the process of expanding its proportional abundance, which is presently at $37 \%$. The TMGMV-N. glauca pathosystem may represent an opportunity to observe competitive exclusion in a natural system over time.

\section{LITERATURE CITED}

1. Bald, J. G., and Goodchild, D. J. 1960. Tobacco mosaic virus in Nicotiana glauca. Phytopathology 50:497-499.

2. Bodaghi, S., Yassi, M. N. A., and Dodds, J. A. 2000. Heterogeneity in the 3 '-terminal untranslated region of tobacco mild green mosaic tobamoviruses from Nicotiana glauca resulting in variants with three or six pseudoknots. J. Gen. Virol. 81:577-586.

3. Dodds, J. A. 1982. Cross protection and interference between electrophoretically distinct strains of cucumber mosaic virus in tomato. Virology 118:235-240.

4. Dodds, J. A. 1993. dsRNA in diagnosis. Pages 273-294 in: Diagnosis of Plant Virus Diseases. R. E. F. Matthews, ed. CRC Press, Boca Raton, FL.

5. Dodds, J. A., Lee, S. Q., and Tiffany, M. 1985. Cross protection between strains of cucumber mosaic virus: Effects of host and type of inoculum on accumulation of virions and double stranded RNA of the challenge virus. Virology 144:301-309.

6. Domingo, E., Escarmis, D., Sevilla, N., Moya, A., Elena, S. F., Quer, J., Novella, I. S., and Holland, J. J. 1996. Basic concepts in RNA virus evolution. FASEB J. 10:859-864.

7. Fraile, A., Escru, F., Aranda, M. A., Malpica, J. M., Gibbs, A. J., and Garcia-Arenal, F. 1997. A century of Tobamovirus evolution in an Australian population of Nicotiana glauca. J. Virol. 71:8316-8320.

8. Gause, G. F. 1971. The Struggle for Existence. Dover Pub., New York.

9. Hall, J. S., French, R., Hein, G. L., Morris, T. J., and Stenger, D. C. 2001. Three distinct mechanisms facilitate genetic isolation of sympatric wheat streak mosaic virus lineages. Virology 282:230-236.

10. Mathews, D. M. 1992. Strains, field studies, and characterization of the $6.8 \mathrm{~K}$ open reading frame of satellite tobacco mosaic virus. Ph.D. diss. University of California, Riverside.

11. McNeil, J. F., French, R., Hein, G. L., Baenziger, P. S., and Eskridge, K. M. 1996. Characterization of genetic variability among natural populations of wheat streak mosaic virus. Phytopathology 86:1222-1227.

12. Palukaitis, P., and Zaitlin, M. 1984. A model to explain the cross protection phenomenon shown by plant viruses and viroids. Pages 213-222 in: Plant Microbe Interactions: Molecular and Genetic Perspectives. T. Kosuge and E. W. Nester, eds. Macmillan, New York.

13. Ponz, F., and Bruening, G. 1986. Mechanisms of resistance to plant viruses. Annu. Rev. Phytopathol. 24:355-381.

14. Randles, J. W., Palukaitis, P., and Davies, C. 1981. Natural distribution, spread, and variation in the tobacco mosaic virus infecting Nicotiana glauca in Australia. Ann. Appl. Biol. 98:109-119.

15. Rodriguez-Cerezo, E., Elena, S. F., Moya, A., and Garcia-Arenal, F. 1991. High genetic stability in natural populations of the plant RNA virus tobacco mild green mosaic virus. J. Mol. Evol. 32:328-332.

16. Sherwood, J. L. 1987. Demonstration of the specific involvement of coat protein in tobacco mosaic virus (TMV) cross protection using a TMV coat protein mutant. J. Phytopathol. 118:358-362.

17. Sherwood, J. L., and Fulton, R. W. 1982. The specific involvement of coat protein in tobacco mosaic virus cross protection. Virology 119:150-158.

18. Solis, I., and Garcia-Arenal, F. 1990. The complete nucleotide sequence of the genomic RNA of the tobamovirus tobacco mild green mosaic virus. Virology 177:553-558. 